\title{
A Systematic Review of Treatment Strategies Including Future Novel Therapies in Crimean-Congo Hemorrhagic Fever
}

\author{
Hamidreza Kouhpayeh ${ }^{1,{ }^{*}}$ \\ ${ }^{1}$ Tropical and Infectious Disease Department, Zahedan University of Medical Sciences, Iran \\ "Corresponding author: Medical Sciences Research Center, Imam Ali Hospital, Zahedan University of Medical Sciences, Zahedan, Iran. Email: hkouhpayeh@yahoo.com
}

Received 2021 January 31; Revised 2021 February 02; Accepted 2021 February 03.

\begin{abstract}
Introduction: Crimean-Congo hemorrhagic fever (CCHF) is an acute fetal illness the case fatality rate (CFR), which without treatment is between $26 \%$ to more than $80 \%$. Despite the administration of ribavirin as a specific antiviral drug for the treatment of CCHF from many years ago, its clinical efficacy is still controversial.

Objectives: This systematic review aimed to evaluate the clinical efficacy of ribavirin, favipiravir, and other treatment options for CCHF, including steroids, immunoglobulin, etc.

Method: This systematic review included 31 articles, three factsheet from WHO, CDC, and ECDC, two editorial letters, and two textbooks from 2002 to 2020. The following databases were searched: Google Scholar, PubMed, Medscape, Cochrane, WHO, CDC, and ECDC.

Results: The selected results of the above articles were concentrated on the different options of supportive treatment, including steroids, immunoglobulin, etc., as well as the efficacy of antiviral drugs, especially ribavirin and favipiravir. While some studies confirmed the clinical efficacy of ribavirin in the treatment of CCHF, some other studies did not confirm its efficacy. All studies justified that supportive therapies are the mainstay of treatment.

Conclusions: The cornerstone of therapy of CCHF is supportive treatment. The clinical efficacy of ribavirin for CCHF treatment is questionable, and further randomized case-control clinical trials are required to confirm and recommend it for CCHF treatment. Also, other treatment strategies, including administration of steroids, immunoglobulin, and monoclonal antibodies (mAbs) require more conclusive data. The promising antiviral drug for CCHF treatment is favipiravir.
\end{abstract}

Keywords: CCHF, Treatment, Ribavirin, Supportive Therapy, Favipiravir

\section{Introduction}

Crimean-Congo hemorrhagic fever (CCHF) was first reported among Russian soldiers in Crimea in 1994, and then it was recognized in the Congo in 1956. CCHF has been distributed in Russia, the Balkans, the Middle East, Central Asia, Western China, and Africa. From 1998 to 2013, it has been more prevalent in Turkey, Russia, Iran, Pakistan, and Afghanistan (1). CCHF is caused by CCHF virus (CCHFV), which is an RNA virus, and its vector is a tick of the genus, Hyalomma. So, it is most commonly transmitted by a tick bite and then by contact with "sheep and cattle" $(1,2)$. Also, contamination with the blood of CCHF patients could ensue nosocomial outbreaks (1).

In Iran, CCHF was first reported in Sarab, Eastern Azerbaijan province, in 1966; but many years before this report, CCHF had been endemic in this region as Ghareh Mikh typhoid (2). Many years later, a new outbreak with 24 cases was reported in Sistan and Baluchistan (SB) province from
1999 to 2004 (3). CCHF is an acute febrile and fetal illness. The case fatality rate (CFR) without treatment is between $26 \%$ to more than $80 \%$ due to severe hemorrhage, circulatory shock, multiple organ failure, and disseminated intravascular coagulation (DIC) (1-4). Early diagnosis and treatment of the disease can significantly reduce the CFR. The mortality rate varies from $0-5 \%$ to greater than $80 \%$ in different regions and medical centers $(1,2,4-6)$.

In vitro and in vivo studies demonstrated that ribavirin is effective in treating CCHF, and it has been used for many years. Some observational studies and the World Health Organization (WHO) have supported its use in the treatment of CCHF; however, many clinical trials have not confirmed the efficacy of ribavirin. In this regard, one review study indicated that there is insufficient evidence to confirm ribavirin efficacy in the treatment of CCHF, and further well-designed clinical trials are required to evaluate its efficacy $(1,7)$. Also, favipiravir is another promising an- 
tiviral drug, which should be evaluated in clinical trials. However, supportive therapy, including administration of fluids and electrolytes, blood, and blood products such as frozen plasma (FFP) and platelets are the mainstay of treatment in $\operatorname{CCHF}(1,6-8)$.

\section{Objectives}

This review aimed to investigate the effectiveness of various components of treatment in CCHF, including specific antiviral drugs.

\section{Method}

This systematic review included 31 articles, three factsheet from the WHO, Centers for Disease Control and Prevention (CDC), and European Centre for Disease Prevention and Control (ECDC), two editorial letters, and two textbooks from 2002 to 2020 . Three of the 34 reviewed articles had been published by the researchers of Zahedan University of Medical Sciences, Iran. The searched databases included Google Scholar, PubMed, Med scope, Cochrane Library, WHO, CDC, and ECDC by keywords including CCHF and treatment. Finally, the abstracts, results, and conclusions of the articles were reviewed.

\section{Results}

The results showed that most studies had evaluated the efficacy of antiviral drugs in CCHF and other therapeutic options, including steroids, immunoglobulin, monoclonal antibodies, immune convalescent sera, and interferon therapy, as well as general supportive therapies for treating CCHF. In addition, these results included novel therapies and future perspectives in CCHF treatment.

Arab-Bafrani et al., in a meta-analysis in 2019, surveyed the results of 24 studies which had no heterogeneity and publication bias to evaluate the crucial parameters in relation to the efficacy of ribavirin in the treatment of CCHF patients. Their results indicated that early administration of ribavirin to CCHF patients was effective in reducing the mortality rate by 1.7 fold, and delay in the administration of this drug could increase the mortality rate by 1.6 fold. They also found that adding corticosteroid to ribavirin for the treatment of CCHF resulted in more reduction in mortality rate by 2.3 folds (9). Finally, they concluded that ribavirin is a crucial antiviral drug that should be used especially in the early stage of disease, and the prescription of corticosteroids along with ribavirin can be effective in hemorrhagic phase with a significant reduction in mortality rate (9).
Saijo (2018) studied the efficacy of specific antiviral drugs on severe fever with thrombocytopenia syndrome (SFTS) due to phlebovirus and reported that ribavirin had inhibitory effects on SFTS virus replication in vitro. Favipiravir inhibited the replication of SFTSV both in vitro and in vivo in interferon alpha receptor (IFNAR-Ko) mice infected with $1 \times 10^{6} 50 \%$ tissue culture infective dose $\left(\mathrm{TCID}_{50}\right)$. The study showed that favipiravir was more effective than ribavirin in the treatment of SFTS patients in Japan. Sanijo described that favipiravir is a promising antiviral drug for SFTS and as a novel therapy for CCHF but mentioned that more case-control RCTs are needed to evaluate its efficacy in SFTS and CCHF patients. Also, supportive therapies including steroid pulse therapy and plasma exchange need further study to evaluate their efficacy in SFTS and CCHF treatment (10). Catherine $\mathrm{H}$. in a review article described that when the oral nucleotide analog drugs such as ribavirin and favipiravir are administered at high doses (150 $\mathrm{mg}$ to $300 \mathrm{mg} / \mathrm{kg}$ ) they may inhibit RNA polymerase of some viruses such as CCHF and Ebola virus in mice and Ebola and Lassa fever viruses in primates. Therefore, favipiravir can be used at high concentrations for antiviral therapy of CCHF and Ebola patients and with ribavirin for treatment of Lassa fever patients; however, favipiravir did not completely inhibit CCHFV in some infected mice at above mentioned doses and some of them died after the treatment (11).

Braira et al., in a systematic review revealed that supportive therapy including the administration of blood products such as thrombocytes, erythrocytes, and fresh frozen plasma in the early stage of disease is an effective strategy to treat CCHE patients. Also, ribavirin is effective in inhibiting CCHFV replication. There may be a synergistic effect of chloroquine or chlorpromazine with ribavirin for combination therapy of CCHF patients. Novel therapy with MxA related to interferon induced GTPase family may interfere with CCHFV replication. They discussed that despite the evidence suggesting that oral ribavirin therapy is effective against CCHF, a recent study did not show its efficacy in reducing mortality rate, so its role in the treatment of CCHF patients is controversial. Specific immunoglobulin against CCHF may be used as a novel therapy for CCHF, but the data about its efficacy is not sufficient (12).

Spengler et al. in a review on the therapeutic intervention in CCHF in 2015, described the antiviral efficacy of ribavirin against CCHFV in cell culture and animal studies, but its clinical efficacy was controversial. The timing of prescription seems to be important, and it is required to be initiated in the early stage of the disease to obtain the best result. Further clinical trials are required to assess the true effectiveness of ribavirin for CCHF. They stated that another RNA virus inhibitor drug, favipiravir, was effective in 
vitro and in vivo against CCHFV (13). Interferon (IFN) had no significant effect on pre-established cell infection with CCHFV; so, its effect for post-exposure treatment of CCHF is unknown. Specific monoclonal antibodies to surface glycoproteins of CCHFV ( $\mathrm{Gn}$ and $\mathrm{Gc}$ ) with neutralization effect in vitro did not necessarily indicate in vivo protection against disease and fatality, which implies a complex interaction between antibody characters and host factors (13).

Intravenous and intramuscular specific immunoglobulin derived from survived CCHF patients were used for CCHF in Soviet Union, South Africa, Turkey, and Bulgaria; but due to the lack of standard controls which prevented producing conclusive results, none of the studies confirmed their efficacy for treatment or prophylaxis of CCHF (13).

A systematic review on CCHF in pregnancy by Yurievna Pshenichnaya et al. in 2017 discussed that there is no specific antiviral therapy for CCHF. The efficacy of ribavirin for the treatment of CHF is controversial due to the different and uncertain results of previous studies. Based on definite teratogenicity of drug in animal species, ribavirin is contraindicated in pregnancy (pregnancy category $\mathrm{x}$ ), but due to high mortality rate of CCHF and risk benefit ratio of drug in pregnancy, it can be used in high-risk patients, especially in the third trimester of pregnancy that the disease is more fatal. Based on their data, 5/13 pregnant women with ribavirin treatment and 15/23 cases without treatment died, even though this difference was not statistically significant. Favipiravir is also contraindicated in pregnancy with more established efficacy in animal model for treatment of $\mathrm{CCHF}$ (14).

In a review article, Leblebicioglu et al. (2012) described the case management and supportive treatment of most patients. In the absence of specific antiviral treatment of CCHF, supportive treatment is the mainstay of treatment. Supportive treatment includes giving intravenous isotonic fluid such as Ringer's lactate solution or normal saline, as well as parenteral nutrition. Blood products including platelet, erythrocyte suspension, and fresh frozen plasma (FFP) may be necessary for patients with massive hemorrhage. In cases with respiratory and renal failure, respiratory support and hemodialysis may be essential for supportive treatment, respectively. Intravenous proton pump inhibitors (PPI) can be given for prevention of gastrointestinal bleeding and oral progesterone for prophylaxis or control of menstrual bleeding. There is not sufficient data to recommend the administration of corticosteroid and intravenous immunoglobulin (IVIG) and plasma exchange for treatment of CCHF (15).

In a review article in 2012, Keshtkar Jahromi et al. reviewed the development of therapy and prophylaxis for CCHF. For this purpose, they reviewed the results of differ- ent studies on different treatment options, and concluded that for supportive therapy of CCHF, nonsteroidal antiinflammatory drugs (NSAIDs) are contraindicated, and intramuscular injection should be avoided. Severe hemorrhage and hypothermia of CCHF cases should be treated with FFP, crystalloid, and colloid infusions (16). They stated that in six observational studies, ribavirin efficacy was unclear for prophylaxis, but it was effective in the treatment of CCHF. Also, four historical comparison studies, three non-randomized case-control RCTs, and three other observational studies in Iran and Turkey showed contradictory results for the efficacy of ribavirin (16). However, in a randomized clinical trial by Koksal et al. (2009), ribavirin did not show any significant effect $(16,17)$. The data for efficacy of immunoglobulin in the treatment and prevention of CCHF is limeted and needs evaluation by RCTs. Monoclonal antibodies (mab) to Gc surface glycoprotein of virus neutralized CCHF, but non-neutralizing mabs to $\mathrm{Gn}$ protected mice from lethal CCHF infection. As a whole conclusion, efficacy of ribavirin remains uncertain and further case control RCTs should be performed to evaluate the effectiveness of ribavirin in the treatment of CCHF. However, they suggested that clinicians should continue to administer ribavirin to suspected cases of CCHF (16).

In a review article by Saijo et al. in 2010 on recent progress in treatment of CCHF and future perspectives of therapy, they described that supportive therapies including maintenance therapies such as hydration and blood transfusion should be commenced as soon as possible. In the case of disseminated intra-vascular coagulation (DIC), treatment is complicated. For CCHF patients with findings which is consistent with acute hemophagocytic syndrome (VAHPS), high dose methylprednisolone had promising effect and should be one choice of therapies. They stated that ribavirin, as a specific antiviral drug, inhibits CCHF replication in vitro and in vivo, and it has been administered orally or intravenously. The results of clinical studies on ribavirin efficacy in the treatment of CCHF are contradictory and there are no conclusive results on its efficacy. However, ribavirin as the only specific antiviral drug in the treatment of CCHF, was recommended to be prescribed as soon as possible (18).

Hematologic and neurologic side effects of ribavirin therapy are common, but severe adverse events were not observed in different studies. Active immunoglobulins against CCHF is derived from convalescent CCHF patients seems to be effective, but due to lack of sufficient data, passive transfer of immunoglobulins is controversial. Further case-control studies are required to determine its efficacy. Saijo et al. explained that interferon $\alpha$ and $\beta$ induced MxA, which belongs to GTPases and inhibits CCHFV replication in vivo in endothelial and hepatoma cells of human due 
to interaction of MxA with nucleocapsid protein of CCHFV. IFN therapy for CCHF was also reported in one paper, which was terminated due to severe adverse effects (18).

According to final report summary of European research results in 2010 - 2015, ribavirin had been approved for treatment of CCHF, but its benefit is still under discussion. Drug T-705 or favipiravir selectively inhibits the replication of CCHFV and is a valid alternative for ribavirin in the treatment of CCHF. CCHF consortium supported the clinical use of favipiravir for treatment of CCHF (19).

In 2013, the WHO described that general supportive therapy is the mainstay of treatment in CCHF patients. Intensive monitoring for in time volume and blood replacement therapy is necessary. Also, they expressed that oral and intravenous ribavirin seems to be effective. Efficacy of immune plasma of survived patients for treatment of CCHF patients has not been demonstrated (20).

According to CDC in 2013, supportive care is the primary treatment for CCHF and includes intensive caring for fluid balance and electrolyte abnormalities, hypoxia, hemodynamic dysfunction, and secondary bacterial infection. CCHFV is sensitive to the antiviral act of ribavirin in vitro and it has some benefits for treatment of CCHF (21).

Jabbari et al. studied the treatment and control strategy in admitted CCHF patients in 2012. They described that treatment of CCHF is primarily supportive and specific therapeutic drugs for CCHF therapy have limitations. Fluid and electrolyte balance, hemodynamic and ventilation support, and mild sedation are the main supportive therapies in the early phase of disease onset. Also, there may be needed to administer blood products such as erythrocytes, platelets, and fresh frozen plasma based on hemostatic situation. Their observation indicated that early prescription of ribavirin to CCHF patients at the first 5 days of illness improves the survival rate, shortens the recovery time, and accelerates the return of laboratory indexes to normal limits. Therefore, treatment with ribavirin is indicated for suspected CCHF cases and post-exposure prophylaxis in health care workers. Prescription of ribavirin along with corticosteroids could be an additional component of the CCHF treatment protocol (22).

Mardani and Jahromi, in a review article on the CCHF in 2007, stated that supportive therapy is the mainstay of CCHF treatment, including maintenance of fluid and electrolyte balance, circulatory volume, and treatment of hypovolemic shock and bacterial infection. They pronounced ribavirin as a guanosine analogue with activity against RNA viruses. Some studies have demonstrated the clinical efficacy of ribavirin for the treatment of CCHF, even though it has not been approved by the Food and Drug Administration (FDA). In the case of outbreaks with high number of CCHF patients, an oral regimen of ribavirin is recommended. In pregnancy, ribavirin is contraindicated; however, in the sever form of CCHF in pregnancy, since the benefits of ribavirin therapy outweigh the fatal risk of drug, it should be recommended. In children, the usage of ribavirin was not approved by the FDA (23).

Three historical cohort studies in Pakistan, Iran, and Turkey indicated the efficacy of ribavirin in terms of fatality and reduced the death rate. However, the study by Ozkurt in 2006 in Turkey demonstrated only the shorter recovery time, but there was not difference in terms of the need for blood and blood products, hospitalization time, and fatality rate between ribavirin-treated and control groups $(23,24)$.

Johnson et al. (2018) investigated the role of ribavirin on CCHF treatment; they included five studies in the systematic review, and 18 studies were excluded due to the high risk of bias. These 18 studies were non-randomized and had not attempted to control the confounding factors, which induced a critical risk of bias. In their statistical analysis, ribavirin was not confirmed in different aspects of reducing mortality, the length of stay in hospital, and needing platelet transfusion even when ribavirin was given in the early phase of CCHF disease. Also, there was not sufficient data for adverse effects of drug and the need to discontinue treatment with ribavirin in CCHF patients. They concluded that reliable evidence to determine the effectiveness of ribavirin is insufficient, and a large placebo controlled RCT is required to evaluate its effect (7).

In a review article by Keshtkar Jahromi in 2014 on CCHF treatment and preventive strategies, it was represented that there was no confirmation for the clinical efficacy of ribavirin, but due to the safety of short duration of treatment with ribavirin and the high CFR of CCHF, it is justified to use ribavirin for treatment of suspected cases in endemic areas. The efficacy of anti-CCHF immunoglobulin or immune sera, as well as monoclonal antibodies specific to Gn and Gc as surface glycoprotein of CCHFV and IVIG have not been approved. High dose of methylprednisolone accelerated improving the platelet and WBC count and Ddimer level in patients with hemophagohistiocytosis. So, these data supported the prescription of high-dose steroid in severe disease, but further studies are required to determine the steroid effect (25).

The ECDC issued a fact sheet about CCHF in 2017 in which stated that there is no validated specific antiviral therapy for CCHF; so, its treatment is dependent on supportive management, including early prescription of FFP, platelet, and erythrocyte preparations. As antiviral drug, ribavirin has been used intravenously or orally with clinical efficacy in some studies, but with no confirmed benefit. Immunoglobulin taken from convalescent patients of CCHF should be further evaluated for treatment (26). 
Oestereich et al. in 2014 evaluated antiviral efficacy of oral ribavirin arbidol and T-705 (favipiravir) on CCHF in a mouse model. They found that favipiravir is highly potent on CCHFV in vitro and in vivo, even its in vivo effect is more than ribavirin for CCHF treatment. Co-administration of both drugs induced benefit rather than side effects (27).

Bodur et al. in 2010 studied the effect of oral ribavirin in 10 patients, and they did not find any effect on the viral load and disease progression in comparison with 40 control patients (28).

Koksal et al. (2009) conducted a prospective randomized cohort study on 136 cases on the efficacy of ribavirin in the treatment of CCHF in the eastern Black Sea region of Turkey country; ribavirin and supportive treatment was given to 64 patients, and 72 patients received only supportive therapy. They concluded that ribavirin had no effect on CCHF prognosis, leukocyte count, and hospitalization duration (17).

Ozkurt et al. in 2005 studied the clinical features and efficacy of ribavirin therapy in 60 admitted cases of CCHF in a case-control study. They found no significant difference in terms of the need for blood and blood products, mean hospitalization period, fatality rate, and hospital expenses between the two groups; however, the recovery time was shorter in the treatment group (24).

In a review article on therapeutic management of CCHF, Fernando et al. (2012) in Spain did not confirm the efficacy of steroid for CCHF treatment in small case series. However, close laboratory monitoring along with fluid and electrolyte balance should be maintained. Platelet, erythrocyte preparations, and FFP are recommended for treatment of hemorrhagic complication. Also, they described that better designed clinical trials must be done to improve the evidence on the efficacy of ribavirin, favipiravir, immunotherapy with serum from convalescent patients, immunoglobulins, monoclonal antibodies, IFN inducible GTPase (MxA), and other experimental drugs such as chloroquine and chlorpromazine. Therefore, they concluded that the cornerstone of CCHF treatment is supportive therapy, but in severe CCHF cases, intravenous ribavirin is justified to be administered. Also, they stated that favipiravir could be a suitable treatment for CCHF, while umifenovir did not show any effect (29).

Mardani, in an editorial report to Archive of SID in 2019, expressed that the overall efficacy of ribavirin for treatment of CCHF was $80 \%$ in confirmed cases and $34 \%$ of suspected cases, while in the systematic review by SoaresWeister et al. the effectiveness of ribavirin for treatment of CCHF was not confirmed. Also, a systematic review and meta-analysis of 24 studies by Arab-Bafrani et al. showed that ribavirin noticeably reduced the mortality rate (by 1.7 fold) (9, 30). Mardani stated that further studies as double-blind randomized clinical trials are required to concentrate on treatment and prevention options, including favipiravir, IVIG, steroids, and mabs (30).

The results of an international multicenter retrospective analysis among research centers in Turkey, Iran, and school of medicine of Johns Hopkins University on ribavirin in the treatment of CCHF in 2016 found that ribavirin did not improve the outcome in CCHF patients (31).

Onder Ergonul, in 2014, stated that the RCTs which did not find any effect for ribavirin were biased due to the inclusion of late cases. Ribavirin may not be effective at the late stage of disease, and the sample size in these RCTs was not calculated. Also, he referred to another biased study in which without the administration of ribavirin the CFR was $5 \%$, while in another study with the early use of ribavirin, the CFR was $2.9 \%$; so, the $5 \%$ fatality rate could not be accepted as a normal rate. Finally, he described that ribavirin has been found to be effective for the treatment of CCHF and as post-exposure prophylaxis (32).

Sharifi-Mood et al. in 2003 studied the efficacy of ribavirin for the treatment of children with CCHF; they evaluated 29 children with confirmed CCHF and treated 25 out of 29 children with oral ribavirin within the first three days of illness onset. The recovery rate was higher in the children who were treated during the initial three days than cases who were treated after this time (84 versus $25 \%$ ). They concluded that oral ribavirin was an effective treatment in children with CCHF (33).

Elaldi et al. in 2007 studied the efficacy of oral ribavirin in confirmed CCHF cases. The survival rate of their treatment and control groups was 93 and 88\%, respectively, which was not statistically significant; therefore, they concluded that ribavirin was not effective (34).

Mardani et al. in 2003 studied the efficacy of oral ribavirin in the treatment of CCHF. They compared the mortality rate of 139 suspected and 69 confirmed cases of CCHF based on oral ribavirin therapy. They found that the efficacy of ribavirin therapy was $80 \%$ among CCHF confirmed cases and 34\% among CCHF suspected cases. They concluded that ribavirin was effective for treating CCHF (35).

\section{Conclusions}

Supportive treatments such as infusion of fluids, electrolytes, and blood products including platelet, erythrocyte preparations, and FFP in the case of bleeding and also intensive monitoring of clinical signs and laboratory data are the mainstay of treatment in CCHF patients. Other treatment options, including immunoglobulin, immune sera from survived patients, mabs against surface glycoproteins of CCHFV, IFN therapy, and high-dose steroid in the case of VAHPS complication seem to be effective, but 
none of them are recommended in the treatment of CCHF due to lack of sufficient and conclusive data. However, further randomized case-control clinical trials are required to determine the efficacy of the abovementioned treatment strategies.

There is still no confirmed specific antiviral drug for the treatment of CCHF. Ribavirin, a guanosine nucleotide, inhibits replication of CCHFV in vitro and in vivo. It has been used orally or intravenously in the treatment of CCHF patients for many years, but the results of many studies in this systematic review on the clinical efficacy of ribavirin in the treatment of CCHF cases were contradictory, and more case-control RCTs are required to confirm the efficacy of ribavirin in the treatment of CCHF.

Favipiravir, another antiviral drug, has been more effective than ribavirin in vivo in mouse model; therefore, it is a promising effective antiviral drug in the treatment of CCHF patients. Currently, ribavirin is justified by WHO, and some studies for the treatment of CCHF suspected cases and can be used in severe forms of CCHF intravenously or orally along with supportive therapies. If supportive treatment is initiated in the early phase of illness onset, it improves the survival rate significantly. Moreover, ribavirin is preferred to be administered in the first three days of disease onset to decrease the mortality rate based on the studies that their results are compatible with the efficacy of ribavirin in the treatment of CCHF.

\section{Footnotes}

Authors' Contribution: The main and correspondent is the same and only author.

Conflict of Interests: It was not declared by the author. Funding/Support: No funding or support.

\section{References}

1. Bennett J, Dolin R, Blaser MJ. Bunya virus hemorrhagic fevers. Mandell, Douglas, and Bennett's principles and practice of infectious diseases. 9th ed. Amsterdam, The Netherlands: Elsevier; 2020.

2. Saebi E. [Crimean hemorrhagic fever]. [Text book of infectious diseases in Iran]. Tehran, Iran: Esmaiel Saebi; 1993. p. 607-16. Persian.

3. Kouhpayeh H. Epidemiologic and clinical and laboratory evaluation of 18 hospitalized cases of Crimean Congo hemorrhagic fever in Bu-Ali Hospital of Zahedan, Iran. Int J Infect Dis. 2002;7:19-22. doi: 10.5812/iji.91707.

4. Kouhpayeh H. An Overview of Complications and Mortality of Crimean-Congo Hemorrhagic Fever. Int J Infect Dis. 2019;6(2):eg1707. doi: 10.5812/iji.91707.

5. Alavi-Naini R, Moghtaderi A, Koohpayeh HR, Sharifi-Mood B, Naderi $\mathrm{M}$, Metanat $\mathrm{M}$, et al. Crimean-Congo hemorrhagic fever in Southeast of Iran. J Infect. 2006;52(5):378-82. doi: 10.1016/j.jinf.2005.07.015. [PubMed: 16182370].

6. Ayatollahi J, Shahcheraghi SH, Mirjalili M. Report of nine cases of Crimean-Congo haemorrhagic fever From Iran. Niger Med J.
2015;56(2):156-9. doi: 10.4103/0300-1652.153409. [PubMed: 25838635] [PubMed Central: PMC4382609].

7. Johnson S, Henschke N, Maayan N, Mills I, Buckley BS, Kakourou A, et al. Ribavirin for treating Crimean Congo haemorrhagic fever. Cochrane Database Syst Rev. 2018;6. CD012713. doi: 10.1002/14651858.CD012713.pub2. [PubMed: 29869797]. [PubMed Central: PMC5994605].

8. Ministry of Health and Modical Education. [Zoonosis]. Tehran, Iran: Ministry of Health and Modical Education; 2021, [cited April 2019]. Persian. Available from: https://health.behdasht.gov.ir/.

9. Arab-Bafrani Z, Jabbari A, Mostakhdem Hashemi M, Arabzadeh AM, Gilanipour A, Mousavi E. Identification of the crucial parameters regarding the efficacy of ribavirin therapy in Crimean-Congo haemorrhagic fever (CCHF) patients: A systematic review and meta-analysis. J Antimicrob Chemother. 2019;74(12):3432-9. doi: 10.1093/jac/dkz328. [PubMed: 31369086].

10. Saijo M. Pathophysiology of severe fever with thrombocytopenia syndrome and development of specific antiviral therapy. $J$ Infect Chemother. 2018;24(10):773-81. doi: 10.1016/j.jiac.2018.07.009. [PubMed: 30098914].

11. Schein $\mathrm{CH}$. Repurposing approved drugs on the pathway to novel therapies. Med Res Rev. 2020;40(2):586-605. doi: 10.1002/med.21627. [PubMed: 31432544]. [PubMed Central: PMC7018532].

12. Wahid B, Altaf S, Naeem N, Ilyas N, Idrees M. Scoping review of Crimean-Congo hemorrhagic fever (CCHF) literature and implications of future research. J Coll Physicians Surg Pak. 2019;29(6):563-73. doi: 10.29271/jcpsp.2019.06.563. [PubMed: 31133158].

13. Spengler JR, Bente DA. Therapeutic intervention in Crimean-Congo hemorrhagic fever: Where are we now? Future Virol. 2015;10(3):2036. doi: 10.2217/fvl.14.115. [PubMed: 26379760]. [PubMed Central: PMC4567545].

14. Pshenichnaya NY, Leblebicioglu H, Bozkurt I, Sannikova IV, Abuova GN, Zhuravlev AS, et al. Crimean-Congo hemorrhagic fever in pregnancy: A systematic review and case series from Russia, Kazakhstan and Turkey. Int J Infect Dis. 2017;58:58-64. doi: 10.1016/j.ijid.2017.02.019. [PubMed: 28249811]. [PubMed Central: PMC5421160].

15. Leblebicioglu H, Bodur H, Dokuzoguz B, Elaldi N, Guner R, Koksal I, et al. Case management and supportive treatment for patients with Crimean-Congo hemorrhagic fever. Vector Borne Zoonotic Dis. 2012;12(9):805-11. doi: 10.1089/vbz.2011.0896. [PubMed: 22607078].

16. Keshtkar-Jahromi M, Kuhn JH, Christova I, Bradfute SB, Jahrling PB, Bavari S. Crimean-Congo hemorrhagic fever: Current and future prospects of vaccines and therapies. Antiviral Res. 2011;90(2):85-92. doi: 10.1016/j.antiviral.2011.02.010. [PubMed: 21362441].

17. Koksal I, Yilmaz G, Aksoy F, Aydin H, Yavuz I, Iskender S, et al. The efficacy of ribavirin in the treatment of Crimean-Congo hemorrhagic fever in Eastern Black Sea region in Turkey. J Clin Virol. 2010;47(1):65-8. doi: 10.1016/j.jcv.2009.11.007. [PubMed:19962342].

18. Saijo M, Morikawa S, Kurane I. Recent progress in the treatment of Crimean-Congo hemorrhagic fever and future perspectives. Future Virology. 2010;5(6):801-9. doi: 10.2217/fvl.10.64.

19. Mirazimi A. Crimean Congo hemorrhagic fever: Modern approaches to diagnostics, surveillance prevention, therapy and preparedness. Luxembourg: Cordis EU Research Results; 2015.

20. World Health Organization. Crimean-Congo haemorrhagic fever. Geneva, Switzerland: World Health Organization; 2014, [cited 2021]. Available from: https://apps.who.int/iris/rest/bitstreams/885423/ retrieve.

21. Centers for Disease Control and Prevention. Crimean-Congo hemorrhagic fever (CCHF). Georgia, USA: Centers for Disease Control and Prevention; 2013, [cited 2021]. Available from: https://www.cdc.gov/vhf/ crimean-congo/index.html.

22. Jabbari A, Tabasi S, Abbasi A, Alijanpour E. Crimean-congo hemorrhagic fever: Treatment and control strategy in admitted patients. Caspian J Intern Med. 2012;3(2):443-4. [PubMed: 24358442]. [PubMed Central: PMC3861911]. 
23. Mardani M, Keshtkar JM. Crimean-Congo hemorrhagic fever. Arch Iranian Med. 2007;10(2):204-14

24. Ozkurt Z, Kiki I, Erol S, Erdem F, Yilmaz N, Parlak M, et al. CrimeanCongo hemorrhagic fever in Eastern Turkey: Clinical features, risk factors and efficacy of ribavirin therapy. J Infect. 2006;52(3):207-15. doi: 10.1016/j.jinf.2005.05.003. [PubMed:15953646].

25. Jahromi MK. Crimean-Congo Hemorrhagic fever-treatment and preventive strategies. Int J Infect Dis. 2014;1(2). e20310. doi: 10.17795/iji20310.

26. European Centre for Disease Prevention and Control. Facts about Crimean-Congo haemorrhagic fever. Solna, Sweden: European Centre for Disease Prevention and Control; 2017, [cited 2021]. Available from: https://www.ecdc.europa.eu/en/crimean-congo-haemorrhagicfever/facts.

27. Oestereich L, Rieger T, Neumann M, Bernreuther C, Lehmann M, Krasemann S, et al. Evaluation of antiviral efficacy of ribavirin, arbidol, and T-705 (favipiravir) in a mouse model for CrimeanCongo hemorrhagic fever. PLoS Negl Trop Dis. 2014;8(5). e2804. doi 10.1371/journal.pntd.0002804. [PubMed: 24786461]. [PubMed Central PMC4006714].

28. Bodur H, Erbay A, Akinci E, Onguru P, Bayazit N, Eren SS, et al. Effect of oral ribavirin treatment on the viral load and disease progression in Crimean-Congo hemorrhagic fever. Int J Infect Dis. 2011;15(1):e44-7. doi: 10.1016/j.ijid.2010.09.009. [PubMed: 21106428].

29. de la Calle-Prieto F, Martin-Quiros A, Trigo E, Mora-Rillo M, Arsuaga M, Diaz-Menendez $\mathrm{M}$, et al. Therapeutic management of Crimean-Congo haemorrhagic fever. Enferm Infecc Microbiol Clin. 2018;36(8):517-22. doi: 10.1016/j.eimc.2017.04.007. [PubMed: 28669587]. [PubMed Central: PMC7103311].

30. Mardani M. Two-decade experience of Crimean-Conge hemorrhagic fever (CCHF) management in Iran. Arch Clin Infect Dis. 2019;14(4). e97887. doi: 10.5812/archcid.97887.

31. Yilmaz G, Sunbul M, Yapar D, Baykam N, Hasanoglu I, Guner R, et al. Ribavirin in treatment of Crimean-Congo hemorrhagic fever (CCHF): An international multicenter retrospective analysis. Open Forum Infect Dis. 2016;3(suppl 1). doi: 10.1093/ofid/ofw172.531.

32. Ergonul O. Evidence supports ribavirin use in Crimean-Congo hemorrhagic fever. Int J Infect Dis. 2014;29:296. doi: 10.1016/j.ijid.2014.08.016. [PubMed: 25461237].

33. Sharifi-Mo B, Alavi-Naini R, Metanat M, Rakhshani F. Ribavirin: An effective drug for treatment of children with Crimean-Congo hemorrhagic fever: A seven-years experience. PakJ Biol Sci. 2006;9(8):1598600. doi:10.3923/pjbs.2006.1598.1600.

34. Elaldi N. Problems in clinical diagnosis and therapy in CCHF: Sivas experience. Istanbul, Turkey: Cumhuriyet University; 2008, [cited 2021]. Available from: https://www.escmid.org/escmid_publications/ escmid_elibrary/material/?mid=1330.

35. Mardani M, Jahromi MK, Naieni KH, Zeinali M. The efficacy of oral ribavirin in the treatment of crimean-congo hemorrhagic fever in Iran. Clin Infect Dis. 2003;36(12):1613-8. doi: 10.1086/375058. [PubMed: 12802764]. 